\title{
ARTICLE Sulforaphane-cysteine-induced apoptosis via phosphorylated ERK1/2-mediated maspin pathway in human non-small cell lung cancer cells
}

\author{
Kai Lin ${ }^{1,3}$, Ronghui Yang ${ }^{1,3}$, Zhongnan Zheng ${ }^{1}$, Yan Zhou ${ }^{1}$, Yang Geng ${ }^{1}$, Yabin $\mathrm{Hu}^{1}$, Sai Wu ${ }^{1}$ and Wei Wu ${ }^{1,2}$
}

Sulforaphane (SFN) was demonstrated to induce apoptosis in a variety of cancers via multiple mechanisms. However, owing to a short half-life in circulation, SFN was not used for clinical treatment yet. Interestingly, SFN analog, sulforaphane-cysteine (SFN-Cys) has a longer half-life in metabolism, and we previously demonstrated that SFN-Cys inhibited invasion in human prostate cancer cells. Here, we would investigate whether SFN-Cys induces apoptosis and find the underlying mechanisms in human non-small cell lung cancer (NSCLC) cells. Western blots were used to test the molecular linkages among extracellular signal-regulated kinases $1 / 2$ (ERK1/2) and downstream signal molecules. Flow cytometry and fluorescence microscopy were used to detect cell death. Cell proliferation assay showed that SFN-Cys inhibited cell viability following a dose-dependent manner. Abnormal cell morphology was viewed after the cells were exposed to SFN-Cys. Flow cytometry showed that SFN-Cys induced cell apoptosis via a dose-dependent manner. Further, SFN-Cys triggered the activation of ERK1/2, which resulted in the upregulation of maspin, Bax, cleaved caspase-3 and downregulation of pro-caspase-3, Bcl-2, $a$-tubulin. Meanwhile, we demonstrated that recombinant caspase-3 cleaved $a$-tubulin in the lysate of cells, which were treated by SFN-Cys. These data indicated that SFN-Cys activated the ERK1/2-mediated mitochondria signaling pathway with maspin upregulation and $a$-tubulin downregulation leading to apoptosis. These findings will help to develop a novel therapeutic to target NSCLC cells.

Cell Death Discovery (2017) 3, 17025; doi:10.1038/cddiscovery.2017.25; published online 3 July 2017

\section{INTRODUCTION}

Mortality of lung cancer worldwide increased yearly. The patients suffered from non-small cell lung carcinoma (NSCLC) had an extremely low 5-year survival rate. ${ }^{1}$ The commonly used therapeutics for NSCLC including surgery, chemotherapy and radiotherapy were not greatly successful because of low efficacy, side effects or drug resistance. ${ }^{2}$ Thus, the development of novel agents to treat NSCLC is not only interesting, but pressing as well.

Epidemiological studies showed that plant-derived drugs, sulforaphane (SFN) and its analogs, have powerful potential to prevent growth of cancer cells. SFN was first found in cruciferous vegetables such as broccoli, brussels sprouts and cabbages, then proved to induce apoptosis during tumorigenesis. ${ }^{3}$ SFN is metabolized in vivo to generate metabolites, such as SFNglutathione, SFN-cysteine-glycine, SFN-cysteine (SFN-Cys), and SFN- $N$-acetylcysteine. ${ }^{4,5}$ SFN metabolites rather than SFN are the major forms of factors in circulation and tissues. SFN-Cys was found to inhibit histone deacetylase activation and have a higher plasma concentration and longer half-life, which might contribute to the inhibition of cancer growth. ${ }^{6}$ However, whether or not SFNCys induced apoptosis in human NSCLC cells and the underlying mechanisms were not clear.

The extracellular signal-regulated kinases 1/2 (ERK1/2) regulate many cellular functions, including proliferation and differentiation. ${ }^{7}$ ERK $1 / 2$ phosphorylation regulates the activation of downstream proteins and signal transduction by various extracellular stimuli. We found that transient ( $<15 \mathrm{~min}$ ) ERK1/2 phosphorylation contributed to cancer proliferation, ${ }^{8}$ whereas sustained ( $>15 \mathrm{~min}$ ) ERK1/2 phosphorylation resulted in growth inhibition and apoptosis. ${ }^{9}$ We found that SFN induced apoptosis by activating ERK $1 / 2$ in human glioblastoma cells, and we just reported that SFN-Cys inhibited invasion by persistent ERK1/2 phosphorylation in human prostate cancer cells. Hence, we proposed that SFN-Cys might induce apoptosis by activating ERK $1 / 2$ and further regulating the downstream signaling molecules in NSCLC cells.

Maspin has been characterized as a suppressor of growth and metastasis influencing many cellular events such as cell motility, adhesion and apoptosis in a variety of cancer cells. ${ }^{10}$ Phosphorylated ERK1/2 might contribute to upregulation of maspin in NSCLC cells. Therefore, it is likely that SFN-Cys might activate ERK1/2 regulating maspin and downstream signaling molecules leading to apoptosis in human NSCLC cells. Bax is a pro-apoptotic protein and $\mathrm{BCl}-2$ is an anti-apoptotic protein. ${ }^{11}$ The interaction between two regulators might result in the increased permeabilization of mitochondrial outer membrane and release of cytochrome $c$ and Smac, subsequently initiating apoptosis. ${ }^{12}$ The ratio of Bax to $\mathrm{BCl}-2$ determines whether cytochrome c can be released and then the caspase family is activated. ${ }^{13}$ The investigation of activated ERK1/2-mediated expression of Bax, $\mathrm{Bcl}-2$ and caspase-3 is necessary to explain the signaling transduction of apoptosis. Apoptotic caspases are sub-categorized initiator caspases, such as

\footnotetext{
${ }^{1}$ Department of Biochemistry and Molecular Biology, School of Basic Medical Sciences, Capital Medical University, Beijing, China and ${ }^{2}$ Institute of Brain Tumor, Beijing Institute for Brain Disorders, Capital Medical University, Beijing, China.

Correspondence: W Wu (weiwu207@ccmu.edu.cn)

${ }^{3}$ These authors contributed equally to this work.

Received 7 February 2017; revised 27 March 2017; accepted 4 April 2017; Edited by A Rufini
} 
caspase-2, caspase-8, caspase-9 and caspase-10 and executioner caspases, such as caspase-3, caspase- 6 and caspase-7. ${ }^{14-17}$ Once initiator caspases are activated, they produce a series of chain reactions, activating several other executioner caspases. ${ }^{15}$ Executioner caspases might degrade over 600 cellular components such as poly (ADP-ribose) polymerase (PARP) in order to induce the morphological changes for apoptosis. ${ }^{18}$ Therefore, we think that SFN-Cys might induce apoptosis through ERK1/2-mediated maspin upregulation, mitochondria death pathway via regulating Bax/Bcl-2 and caspase-3. Meanwhile, cell skeletal protein $a$-tubulin is also a substrate of caspase-3, and plays a key role in cell growth, migration and nutrient transfer. ${ }^{16}$ Microtubules are constituted by $\alpha$-tubulin and $\beta$-tubulin. ${ }^{19}$ They maintain dynamic balance of microtubule via polymerization and depolymerization. Once $a$-tubulin is disturbed, the dynamic balance is broken and apoptosis occurs. ${ }^{20}$ Investigation of SFN-Cys-induced $a$-tubulin downregulation is beneficial to find new mechanisms to elucidate SFN-Cys-induced apoptosis.

In summary, here we will investigate and find some novel mechanisms involved in SFN-Cys-induced apoptosis in NSCLC cells. These studies will assist us to develop new anticancer drugs with lower toxicity and resistance, higher safety and efficiency to treat cancer patients.

\section{RESULTS}

SFN-Cys inhibits cell proliferation in a dose-dependent manner Cells were treated with increasing concentrations of SFN-Cys $(0,10,20,30,40,50,60,70$ and $80 \mu \mathrm{M})$ for $24 \mathrm{~h}$. The results showed that cell viability was decreased significantly after the cells were treated by SFN-Cys for $24 \mathrm{~h}$ in a dose-dependent manner (Figures 1a and b), indicating that SFN-Cys inhibited cell growth in those two cell types. In particular, the decreasing trends of cell viability in A549 cells are greater than those in SK-1 cells. More, the significant reduction of cell viability started at the dose of $20 \mu \mathrm{M}$. Therefore, $20 \mu \mathrm{M}$ should be the optimal treatment concentration of SFN-Cys for the further studies.

\section{SFN-Cys changed cell morphology}

Morphological observation showed that the cells treated with SFN-Cys were round and transparent with short pseudopodia (Figures $1 \mathrm{c}$ and $\mathrm{d}$ ). With increasing concentrations, number of the dead cells increased. At the concentration of $20 \mu \mathrm{M}$, there was an obvious cell death in A549 and SK-1 cells and simultaneously morphological changes were observed. These alterations of cell morphology might result from the inhibition of cell growth and apoptosis.

\section{SFN-Cys induced apoptosis in a dose-dependent manner}

To confirm that SFN-Cys-induced inhibitory effect on NSCLC cells was due to cell apoptosis, flow cytometry assay was performed to verify and quantify the percentages of apoptotic cells. The percentages of the early and late apoptotic cells induced by SFN-Cys were represented in the lower right (LR) and upper right (UR) quadrant of the flow cytometry histograms, respectively. The total percentages of apoptotic A549 cells (UR+LR) increased in SFN-Cys-treated cells (10 $\mu \mathrm{M}$ SFN-Cys, 3.4\%; $20 \mu \mathrm{M}$ SFN-Cys, 5.8\%; $30 \mu \mathrm{M}$ SFN-Cys, 10.8\%), compared with non-treated cells (3.2\%) for $24 \mathrm{~h}$ (Figure 2a). Similar data were observed in SK-1 cells, where the total percentages of apoptotic cells increased from $12.2 \%$ in control cells to $13.0 \%, 21.6 \%$ and $37.9 \%$ in cells treated with 10,20 and $30 \mu \mathrm{M}$ SFN-Cys, respectively (Figure $2 \mathrm{~b}$ ). The results showed that treatment with 10, 20 and $30 \mu \mathrm{M}$ SFN-Cys for $24 \mathrm{~h}$ induced apoptosis in A549 and SK-1 cells in a dose-dependent manner. The results indicated that SFN-Cys exerted an anticancer effect on NSCLC cells.
Further, combined with PD98059, SFN-Cys-induced apoptosis was reversed in both cell types (Figures $2 c$ and d). These data suggested that phosphorylated ERK1/2 contributed to SFN-Cysinduced apoptosis.

SFN-Cys-induced apoptosis via sustained ERK1/2 phosphorylation Here we investigated whether the SFN-Cys-induced apoptosis of A549 and SK-1 cells was associated with the intracellular signaling pathways. Previous studies showed that phosphorylation of ERK $1 / 2$ reached the peak at $24 \mathrm{~h}^{21}$ Therefore, we chose $24 \mathrm{~h}$ as the optimal time for subsequent study. The cells were treated with increasing doses of SFN-Cys $(0,10,20$ and $30 \mu \mathrm{M})$ for $24 \mathrm{~h}$. Western blot demonstrated that phosphorylation of ERK1/2 was significantly enhanced with the increased drug concentrations (Figure 3a). The results showed that SFN-Cys phosphorylated ERK1/2 via a sustained manner in both A549 and SK-1 cells. To examine whether PD98059 weakens SFN-Cys-induced inhibition in NSCLC cells, cells were cultured in the presence of either SFN-Cys $(20 \mu \mathrm{M})$ or PD98059 $(25 \mu \mathrm{M})$, respectively, or a combination of the two. The results revealed that the phosphorylation of ERK $1 / 2$ was significantly decreased in the cells treated with both SFN-Cys $(20 \mu \mathrm{M})$ and PD98059 $(25 \mu \mathrm{M})$ compared with the cells treated with SFN-Cys only (Figure 3b).

\section{SFN-Cys upregulated maspin via activating ERK1/2}

Western blot analysis showed that maspin was upregulated in a dose-dependent manner (Figure 3c). Either SFN-Cys (20 $\mu \mathrm{M})$ or PD98059 $(25 \mu \mathrm{M})$, respectively, or a combination of the two were used to test the expression of maspin. Western blot showed that PD98059 diminished the upregulation of maspin (Figure 3d). These data indicated that activated ERK $1 / 2$ contributed to maspin upregulation.

SFN-Cys upregulated Bax and downregulated $\mathrm{Bcl}-2$ via sustained ERK1/2 phosphorylation

Western blot showed that SFN-Cys upregulated Bax expression (Figure 4a). The cells were treated with PD98059 to verify whether SFN-Cys regulated Bax via ERK1/2 phosphorylation. As a result, western blot showed that PD98059 was able to reverse the upregulation of Bax triggered by SFN-Cys (Figure 4b). These results indicated that SFN-Cys markedly upregulated Bax by phosphorylating ERK1/2. Inhibition of ERK1/2 activation through PD98059 blockade weakened the upregulation of Bax expression. Similarly, western blot showed that SFN-Cys downregulated $\mathrm{BCl}-2$ (Figure 4c). Furthermore, the cells were pretreated with PD98059 to verify whether this regulation of $\mathrm{Bcl}-2$ resulted from the phosphorylation of ERK1/2. Significant changes were found between SFN-Cys-only and PD98059 plus SFN-Cys group, suggesting that blockade of ERK1/2 phosphorylation eradicated inhibition of $\mathrm{BCl}-2$ expression (Figure $4 \mathrm{~d}$ ). Thus, activated ERK1/2 might regulate $\mathrm{Bax} / \mathrm{BCl}-2$ ratio leading to cell death in response to SFNCys.

SFN-Cys-induced activation of caspase-3 and downregulated $a$-tubulin via activating ERK1/2

The cells were treated with increasing doses of SFN-Cys $(0,10,20$ and $30 \mu \mathrm{M}$ ) for $24 \mathrm{~h}$. Western blot showed that pro-caspase-3 was significantly decreased, whereas cleaved caspase-3 was significantly increased with the rising SFN-Cys concentrations in A549 and SK-1 cells (Figures $5 \mathrm{a}$ and b). In addition, we detected the expression of $a$-tubulin in the cells in response to SFN-Cys. Results showed that $a$-tubulin was significantly decreased with the increased drug concentrations (Figure 5c). Meanwhile, western blot showed that PD98059 reversed the downregulation of $a$-tubulin induced by SFN-Cys (Figure $5 \mathrm{~d}$ ). We also demonstrated that $a$-tubulin was cleaved by recombinant caspase- 3 in the lysate 
a

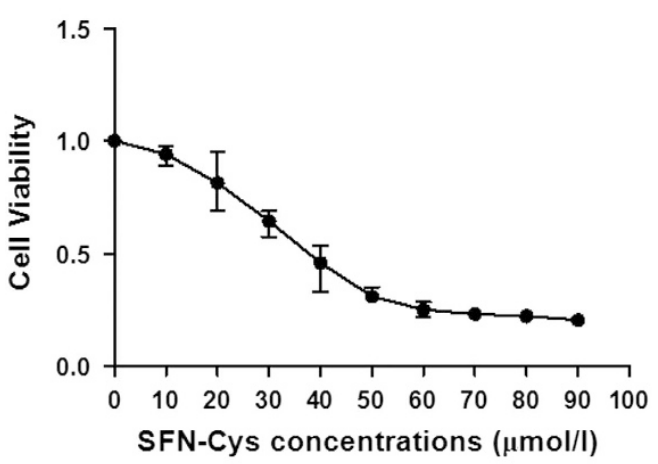

b

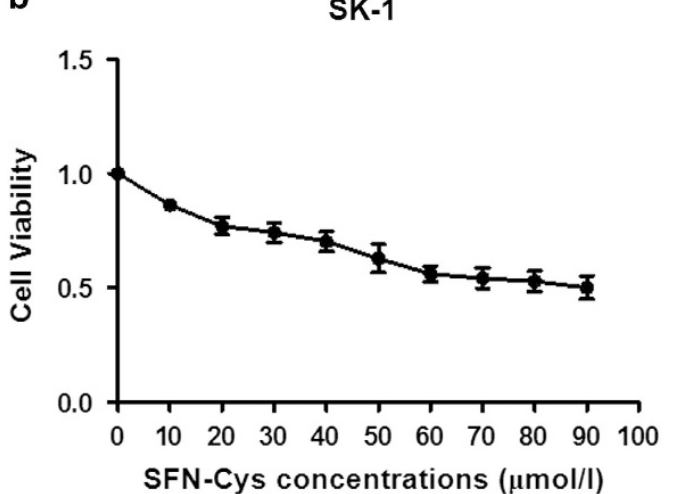

C
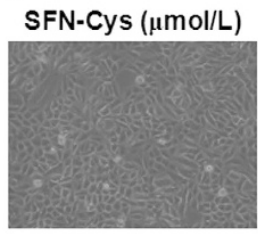

0

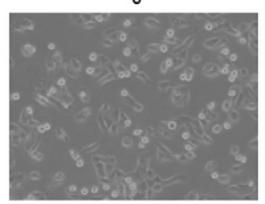

30

d

SFN-Cys $(\mu \mathrm{mol} / \mathrm{L})$

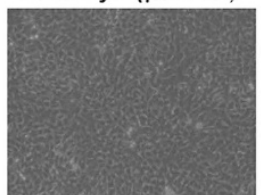

0

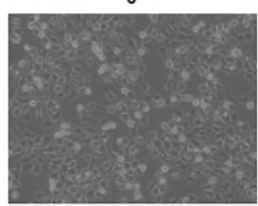

30
A549

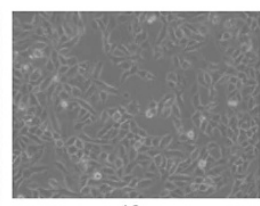

10

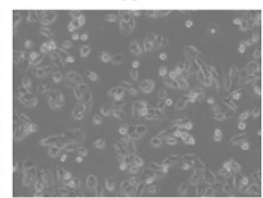

40

SK-1

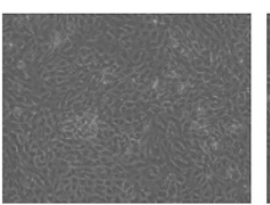

10

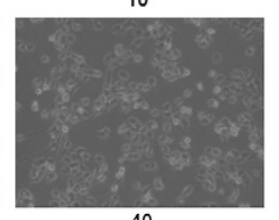

40

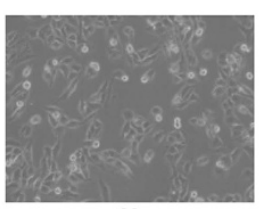

20

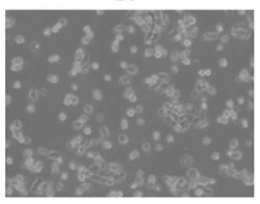

50

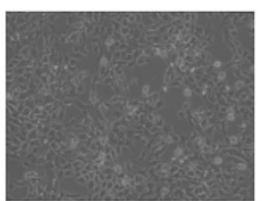

20

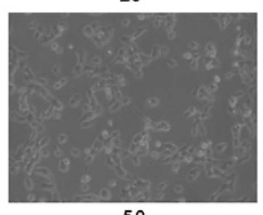

50

Figure 1. Cell viability analysis and morphological changes after treated with SFN-Cys. (a) A549 and (b) SK-1 cells were treated with various concentrations of SFN-Cys $(0,10,20,30,40,50,60,70$ and $80 \mu \mathrm{M})$ for $24 \mathrm{~h}$. SFN-Cys significantly inhibited the proliferation of A549 and SK-1 cells in a dose-dependent manner. These results are presented as mean \pm S.D. from three independent experiments. Error bars indicate uncertainty errors in graphs to relay $95 \%$ of confidence in the interpreted data. The (c) A549 and (d) SK-1 cells were examined with Leica DMIRB Microscope at $\times 100$ magnification. Cells treated with various concentrations of SFN-Cys $(0,10,20,30,40$ and $50 \mu \mathrm{M})$ generated morphological changes.

of cells, which were treated with SFN-Cys in A549 and SK-1 cells (Figure $5 \mathrm{e}$ ). We further detected the expression of $a$-tubulin in A549 and SK-1 cells by immunofluorescence. The results showed that SFN-Cys $(20 \mu \mathrm{M})$ treatment caused shorter cellular pseudopodia, and the level of a-tubulin was markedly reduced (Figure 6a). These results indicated that SFN-Cys might induce activation of caspase- 3 leading to degradation of $\alpha$-tubulin and apoptosis in A549 and SK-1 cells.

\section{DISCUSSION}

SFN is a potent chemopreventive agent inducing apoptosis in a variety of tumor cells. ${ }^{22}$ However, owing to the short half-life in circulation, SFN has not been used in the clinical treatment although a few clinical trials were done. However, SFN-Cys, as a major metabolite of SFN, was found to have extensive tissue distribution in the lung of treated mice and a longer half-life in circulation. $^{23}$ We reported that SFN-Cys inhibited invasion in prostate cancer, ${ }^{24}$ but we do not know if it also inhibits cell growth in lung cancer cells. Here we evaluated its function and working mechanisms causing apoptosis in NSCLC cells. The results showed that SFN-Cys inhibited cell proliferation in A549 and SK-1 cells, which also provided an optimal concentration and treatment time to study apoptosis in vitro. Further, SFN-Cys phosphorylated ERK1/2 leading to apoptosis, which might result from the upregulation of maspin, Bax, cleaved caspase-3, and downregulation of $\mathrm{Bcl}-2$, pro-caspase- 3 and $a$-tubulin. Furthermore, recombinant caspase- 3 cleaved $a$-tubulin in the cells treated with
SFN-Cys. These findings revealed a novel mechanism, which SFNCys exhibited its pro-apoptotic effect in NSCLC cells (Figure 6b).

In the present model, we demonstrated that SFN-Cys inhibited cell proliferation and induced cell apoptosis in both A549 and SK-1 cells in a dose-dependent way. The cell viability showed a remarkable decrease and the apoptotic rates increased significantly following the treatment with $20 \mu \mathrm{M}$ SFN-Cys. The cell morphological changes with round shapes and shorter pseudopodia also indicated that SFN-Cys promoted apoptosis and inhibited invasion in human NSCLC cells. As a result of this, we further investigated the molecular mechanisms of SFN-Cysmediated apoptosis. We found that SFN-Cys significantly increased ERK1/2 phosphorylation in a dose-dependent manner. However, the phosphorylated ERK1/2 inhibitor, PD98059 significantly reversed SFN-Cys-induced apoptosis. These indicated that SFN-Cys induced apoptosis through sustained ERK1/2 activation in human NSCLC cells.

ERK $1 / 2$ is the major kinase that plays a key role in numerous signaling pathways, and is often activated in cancer cells. ${ }^{25}$ Sustained activation of ERK $1 / 2$ contributed to apoptosis, and acute activation of ERK1/2 caused tumor initiation and progression. ${ }^{26}$ The ERK $1 / 2$ activation contributed to intracellular protein-protein interactions and the regulation of multiple cellular processes. Our previous studies demonstrated that SFN-Cys acted as a chemopreventive agent through activating ERK1/2 in human prostate cancer DU145 and PC3 cells, a few downstream effectors were involved. ${ }^{24}$ However, the linkage between ERK1/2 phosphorylation and the modulation of maspin on cell apoptosis has not been reported yet. Maspin is a tumor-suppressor protein, and its 

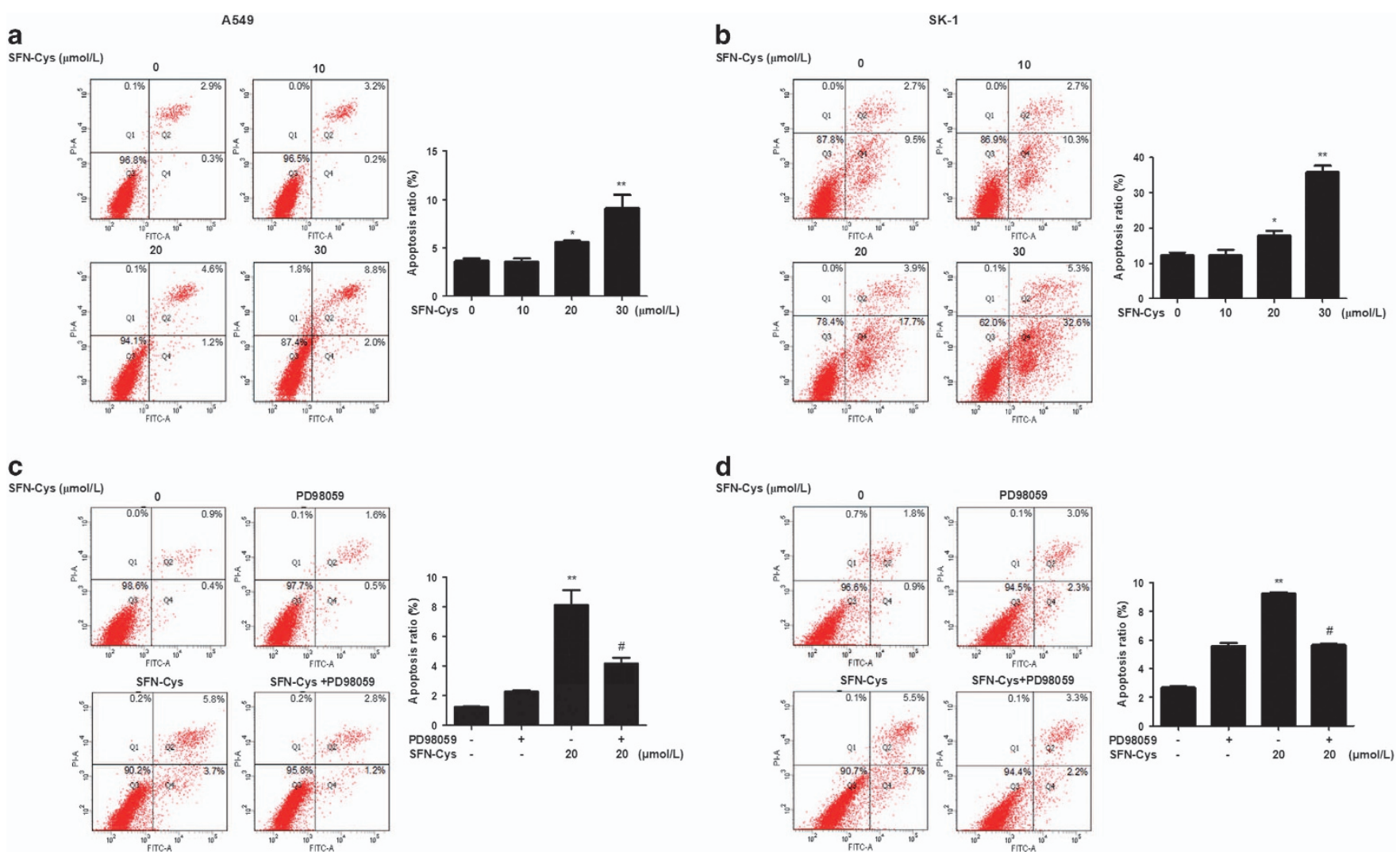

Figure 2. SFN-Cys-induced dose-dependent apoptosis in human A549 and SK-1 cells. (a) A549 and (b) SK-1 cells were treated with various concentrations of SFN-Cys $(0,10,20$ and $30 \mu \mathrm{M})$ for $24 \mathrm{~h}$, and stained with Annexin V-FITC/PI. Compared with the control cells, flow cytometry revealed that the apoptotic rates of the SFN-Cys treated (c) A549 and (d) SK-1 cells were significantly increased $(P<0.01)$, whereas the apoptotic rates of the SFN-Cys-treated cells combined with PD98059 was declined $(P<0.05)$. These results represented three independent tests. Error bars indicate uncertainty errors in graphs to relay $95 \%$ of confidence in the interpreted data. ${ }^{*}$ Indicates $P<0.05$ versus the control group. ${ }^{* *}$ Indicates $P<0.01$ versus control group. " Indicates $P<0.05$ versus the SFN-Cys-only group.

overexpression might induce apoptosis through regulating several members of $\mathrm{BCl}-2$ family. ${ }^{27}$ Interestingly, our results showed that SFN-Cys significantly increased the expression of maspin in NSCLC cells in a dose-dependent manner, suggesting that SFN-Cysinduced apoptosis might be associated with maspin upregulation. Particularly, once the SFN-Cys-treated cells were exposed to PD98059, the upregulation of maspin was reversed, suggesting that phosphorylated ERK1/2 upregulated maspin leading to apoptosis.

Maspin is the inducer of apoptosis via attaching to extracellular matrices, increasing sensitivity of apoptosis and inhibiting angiogenesis. ${ }^{28}$ Further, it is reported that the increased apoptosis in maspin-expressing cells lead to the changes in the protein levels of $\mathrm{BCl}-2$ family. ${ }^{29}$ Maspin linked to the upregulation of the pro-apoptotic protein Bax, which was translocated into the mitochondria during apoptosis. ${ }^{30} \mathrm{Bcl}-2$ is a member of the antiapoptotic Bcl-2 family, which is involved in stabilizing mitochondrial membrane integrity. ${ }^{31}$ More studies have elaborated that $\mathrm{BCl}-$ 2 preserves the mitochondrial membrane and inhibits the release of internal calcium into the cytoplasm, whereas Bax is processed on the outer mitochondrial membrane and regulates the release of cytochrome $\mathrm{c}^{17,31}$ The reasons are that $\mathrm{Bcl}-2$ family proteins activated the intrinsic apoptotic pathway causing mitochondrial cytochrome $c$ to be released into the cytosol. ${ }^{12}$ This molecule might bind an adaptor protein APAF-1, which recruits initiator caspase-9. ${ }^{32}$ Owing to the changed level of $\mathrm{Bcl}-2$ family proteins, the apoptotic pathway became more active in maspin-expressing cells and this leads to the formation of a caspase activating multiprotein complex called the apoptosome, which accelerates apoptosis. $^{33}$
Cell apoptosis is mainly induced by caspases, a family of cysteine aspartyl-specific proteases. ${ }^{34}$ Once activated, initiator caspases such as caspase- 9 will cleave and activate other executioner caspases such as caspase- 3 and caspase-7. This leads to degradation of cellular components for apoptosis. ${ }^{17}$ The caspase- 3 and caspase-7 might in turn cleave a large amount of cellular substrates, such as PARP, ${ }^{18}$ which acts on mitochondria and activates cell death process through mitochondrial depolarization/membrane permeability transition and release of cytochrome $c$, apoptosis-inducing factor or endonuclease $G$ into the cytosol. ${ }^{35}$ The previous studies showed that caspase-3 could cleave PARP to obtain the ability of detecting and repairing DNA damages, and then leading to cell apoptosis. ${ }^{18}$ Hence, we inferred that SFN-Cys-induced cell apoptosis might be involved in an intrinsic pathway.

In addition, we proved that caspase- 3 cleaved $a$-tubulin, which is a vital component of cell microtubule. As a structural element of cell skeleton, microtubule supports cell structure and function stabilization, whereas mitotic spindles in eukaryotic cells segregate their chromosomes during cell division. ${ }^{36}$ Minor changes in microtubule might contribute to cell death; when $a$-tubulin was modified with tyrosination and acetylation, microtubules maintain a dynamic balance. ${ }^{37}$ Once the balance was destroyed, the microtubule structure broke up, apoptosis occurred.

In summary, our results revealed that SFN-Cys with longer halflife in circulation induced apoptosis in A549 and SK-1 cells via persistently activated ERK1/2, upregulating maspin and Bax, and downregulating $\mathrm{Bcl}-2$ and $a$-tubulin, ultimately leading to the activation of caspase- 3 in NSCLC cells. Thus, the present findings provided a new mechanism, which SFN-Cys induced apoptosis, 
a
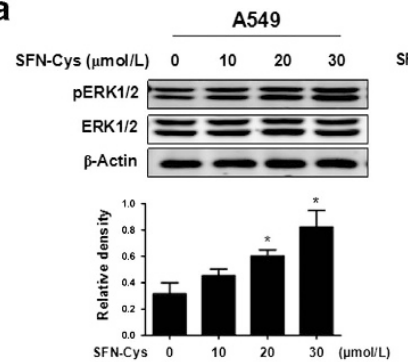

b
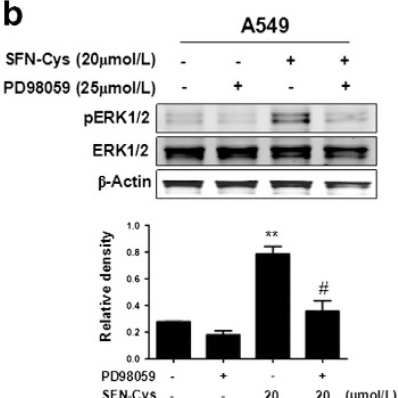

SK-1
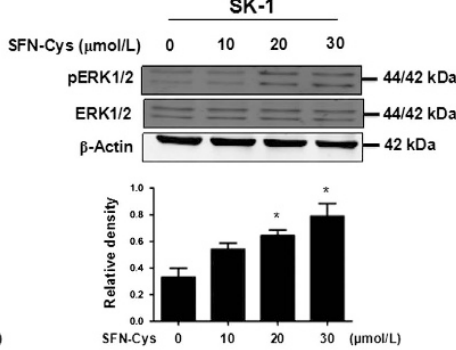

C
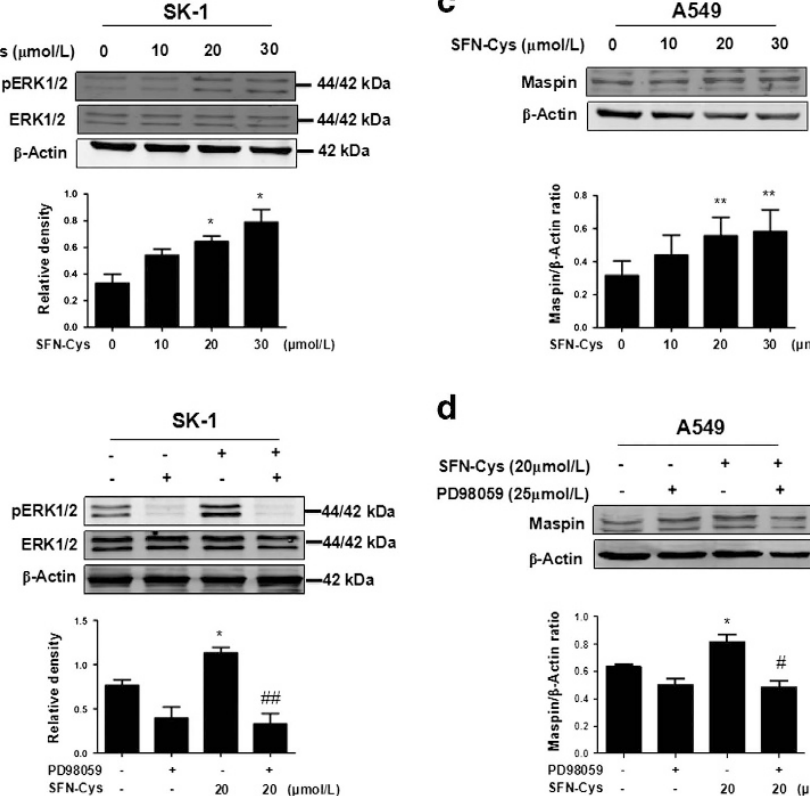

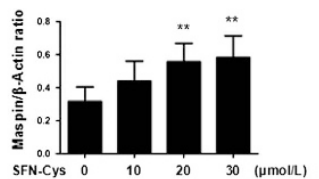

d
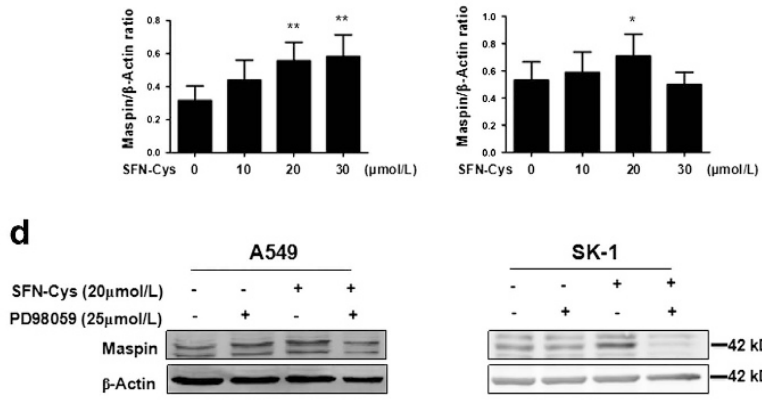

Figure 3. (a) Western blot analysis of the expression of ERK1/2 and pERK1/2 in A549 and SK-1 cells following treated with SFN-Cys (0, 10,20 and $30 \mu \mathrm{M}$ ) for $24 \mathrm{~h}$. (b) A549 and SK-1 cells were cultured with $20 \mu \mathrm{M}$ SFN-Cys, $25 \mu \mathrm{M}$ PD98059, or a combination of the two compounds for $24 \mathrm{~h}$, and the expression levels of ERK $1 / 2$ and pERK1/2 were analyzed. (c) Western blot was used to analyze the expression of maspin following treated with different concentrations of SFN-Cys $(0,10,20$ and $30 \mu \mathrm{M})$. (d) The expression of maspin following treated with SFN-Cys and PD98059 alone or in combination. After treatment with PD98059 $(25 \mu \mathrm{M})$ for 30 min, the cells were incubated with $20 \mu \mathrm{M}$ SFN-Cys for 24 h. The upregulation of maspin induced by SFN-Cys was reversed by PD98059. $\beta$-Actin was used as a control. These results were represented as the mean \pm S.D. of three independent tests. Error bars indicate uncertainty errors in graphs to relay $95 \%$ of confidence in the interpreted data. ${ }^{*}$ Indicates $P<0.05$ versus the control group. ${ }^{* *}$ Indicates $P<0.01$ versus control group. ${ }^{\#}$ Indicates $P<0.05$ versus the SFN-Cys-only group.

$\# \#$ Indicates $P<0.01$ versus SFN-Cys-only group.

a
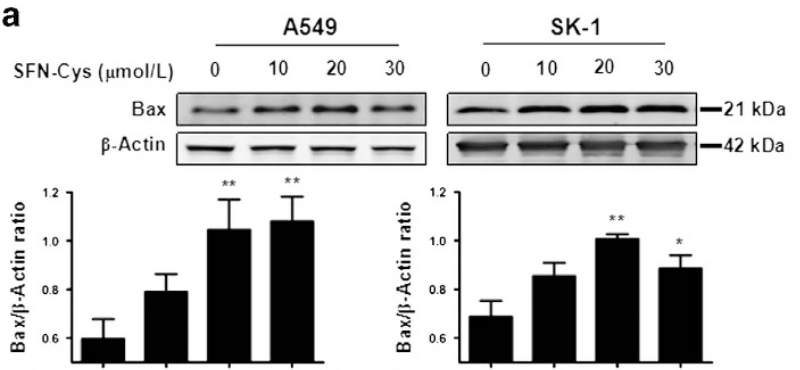

C
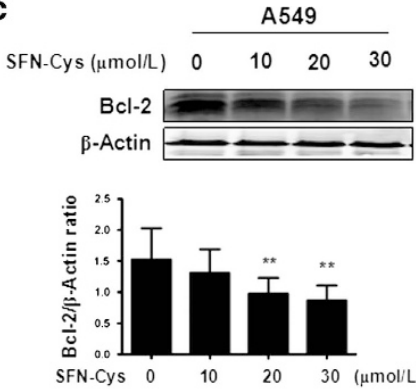

d

b

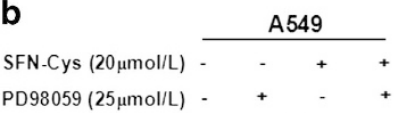
-Actin
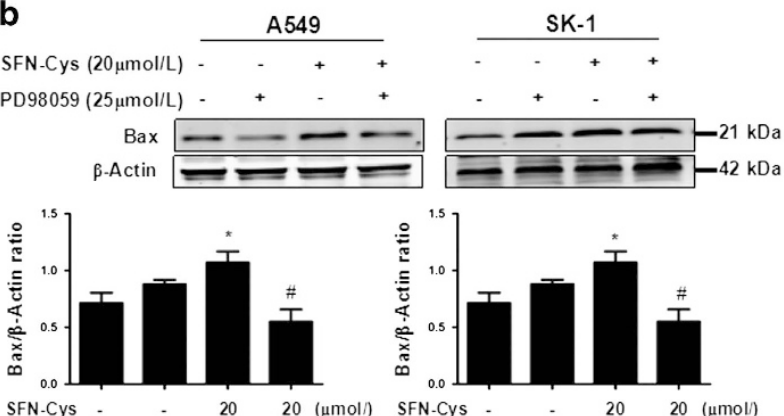

SFN-Cys
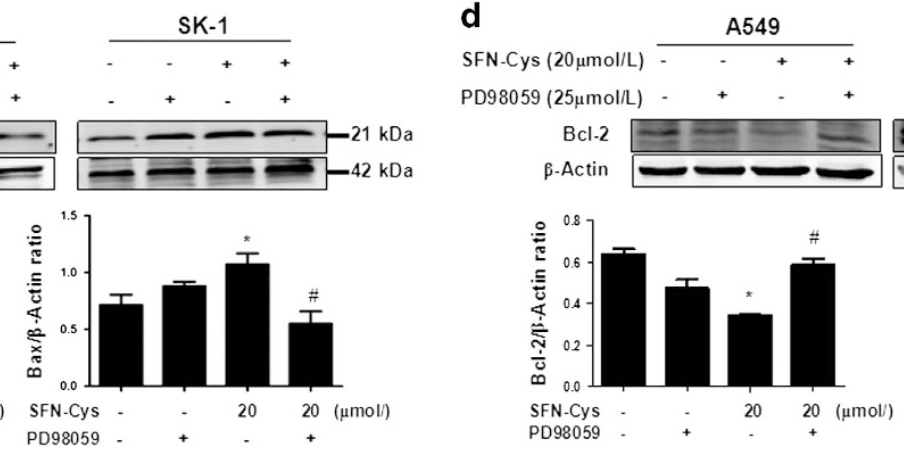
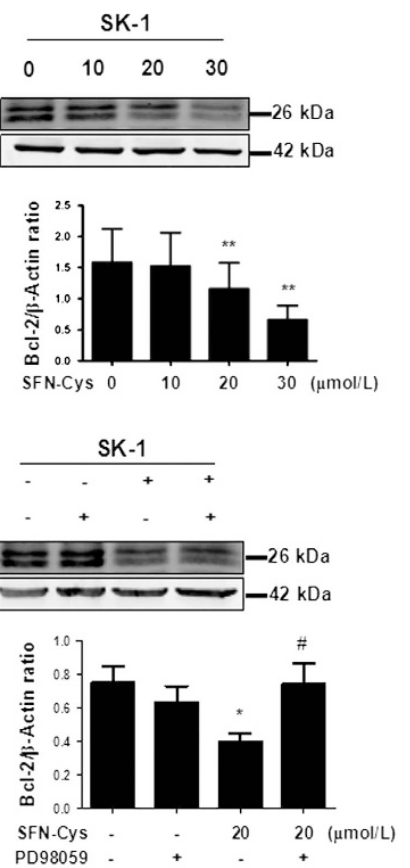

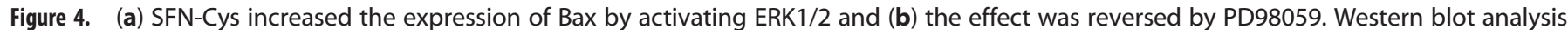

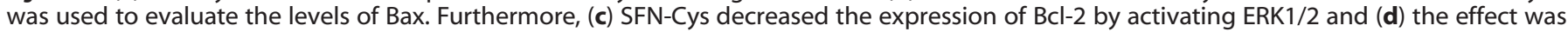

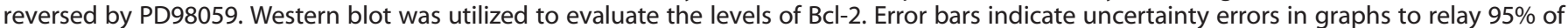
confidence in the interpreted data. * Indicates $P<0.05$ versus control group. ${ }^{* *}$ Indicates $P<0.01$ versus control group. ${ }^{\#}$ Indicates $P<0.05$ versus SFN-Cys only group. 
a
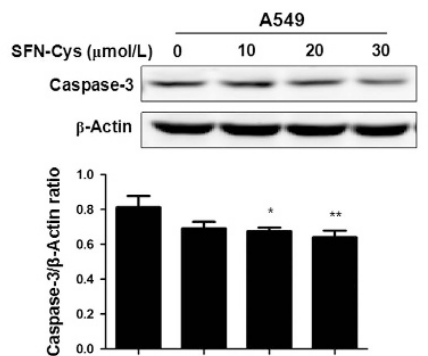

SFN-Cys 0
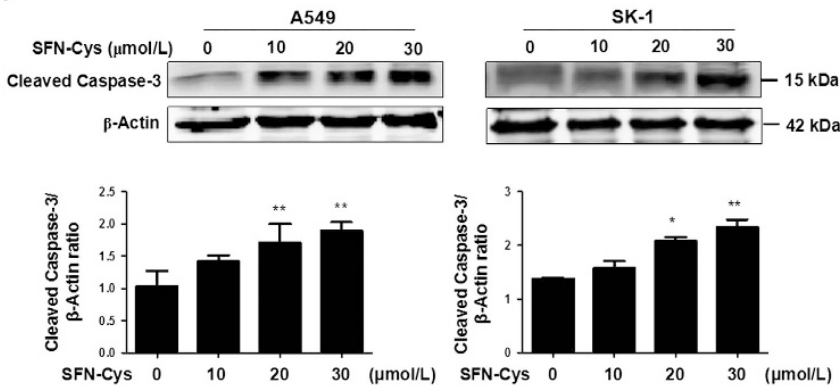

e

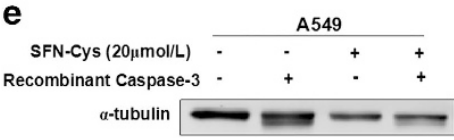

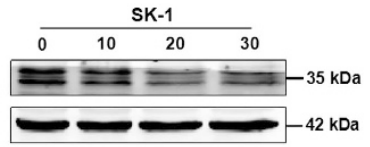

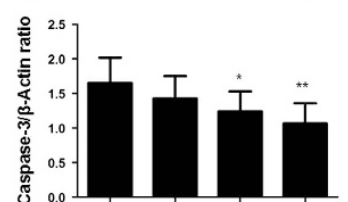

$30(\mu \mathrm{mol} / \mathrm{L})$
C
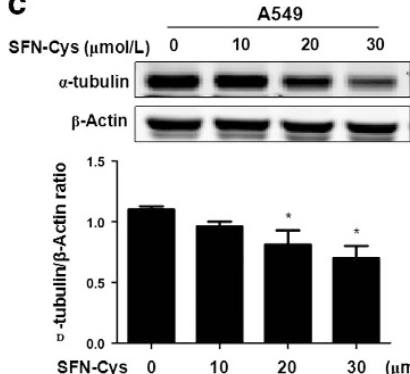

$\begin{array}{lllllll}\text { SFN-Cys } & 0 & 10 & 20 & 30 & \text { ( } \mu \text { mol/L) SFN-Cys } 0\end{array}$
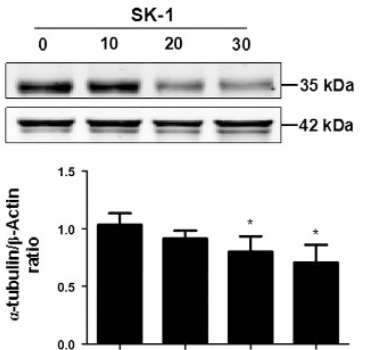

$\begin{array}{llll}10 & 20 & 30 & (\mu \mathrm{mol} / \mathrm{L})\end{array}$

\section{d}
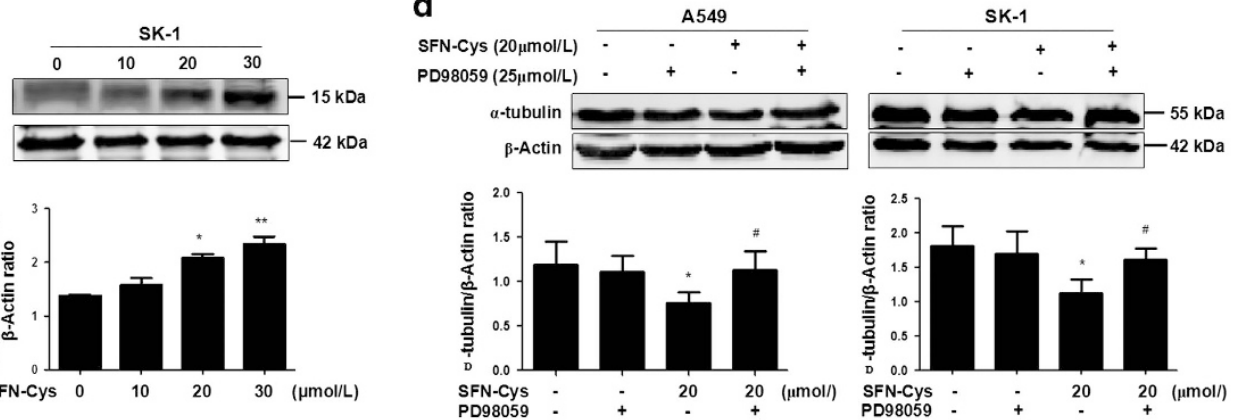

Figure 5. Western blot was utilized to evaluate the levels of pro-caspase-3, cleaved caspase-3 and $a$-tubulin. (a) SFN-Cys downregulated the level of pro-caspase-3. (b) SFN-Cys upregulated the expression of cleaved caspase-3 via increased concentrations; (c) and (d) SFN-Cys decreased $a$-tubulin, whereas PD98059 reserved this process. (e) In vitro, recombinant caspase-3 cleaved $a$-tubulin to produce a band of $53 \mathrm{kDa}$, suggesting that SFN-Cys activated caspase-3 might degrade $a$-tubulin. Each experiment was performed in triplicate. Error bars indicate uncertainty errors in graphs to relay $95 \%$ of confidence in the interpreted data. ${ }^{*}$ Indicates $P<0.05$ versus control group. ${ }^{* *}$ Indicates $P<0.01$ versus control group. ${ }^{\#}$ Indicates $P<0.05$ versus SFN-Cys only group.

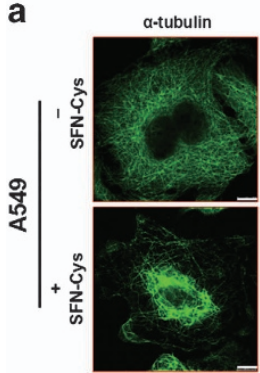

a-tubulin

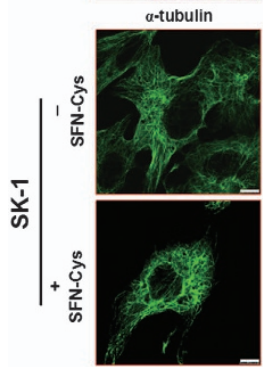

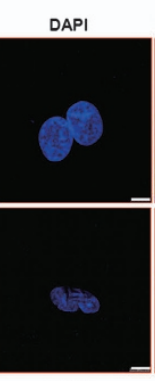

DAPI

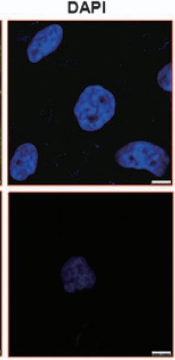

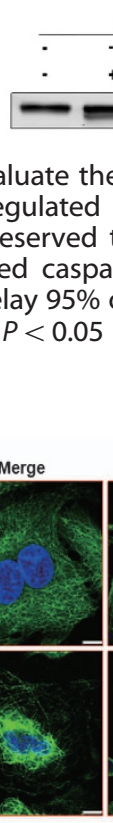

Merge

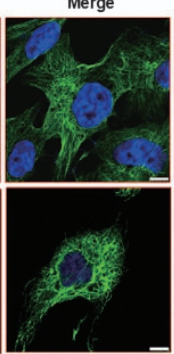

Merge

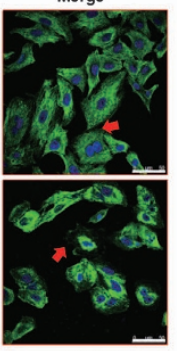

Merge

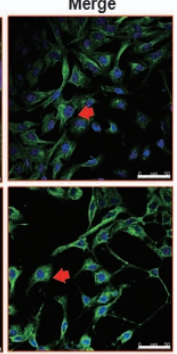

b

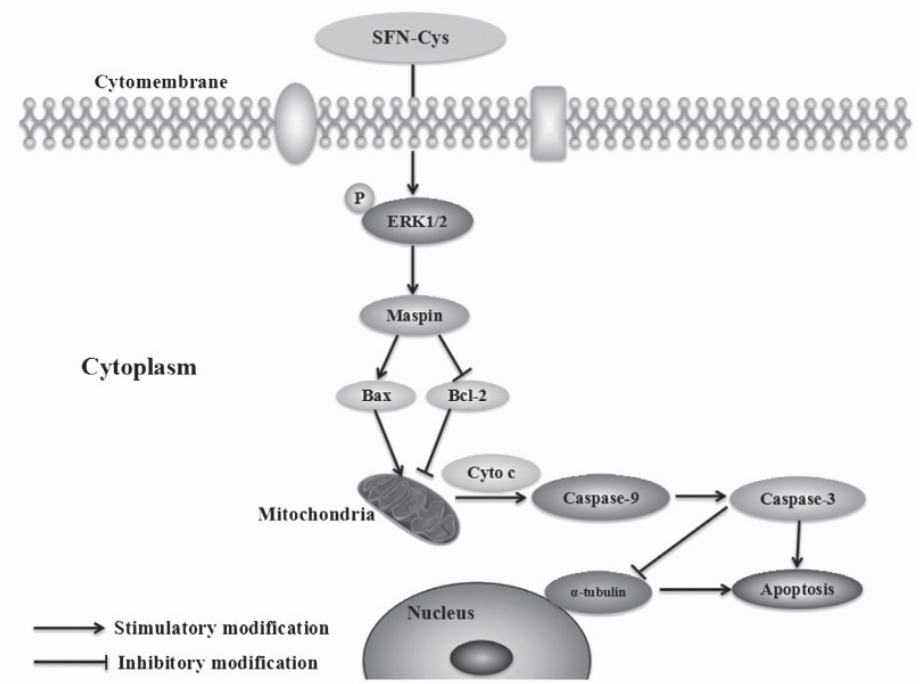

Figure 6. (a) The cells were treated with $20 \mu \mathrm{M}$ of SFN-Cys for $24 \mathrm{~h}$ and measured by laser scanning confocal microscopy. The cell nucleus was dyed purple and $a$-tubulin was colored green. Immunofluorescence showed that $a$-tubulin got fuzzy and loose arrangement in the treated cells compared with the normal cells. a-Tubulin expression was downregulated in response to SFN-Cys. All of the experimental values were determined via identical instrument settings to allow for a quantitative comparison of the cell-associated fluorescent intensity values. Scale bars, 7.5 and $50 \mu \mathrm{m}$. (b) Assumed signaling pathway map for SFN-Cys-induced apoptosis in human NSCLC cells. 
suggesting that we might develop a novel therapeutic to treat human NSCLC.

\section{MATERIALS AND METHODS}

Reagents

SFN-Cys was the product of Santa Cruz Biotechnology (Santa Cruz, CA, USA). BCA protein assay kit was purchased from Invitrogen (Carlsbad, CA, USA). Annexin V-FITC apoptosis assay kit was purchased from GenStar (Beijing, China). Antibody against caspase-3 $(1: 200)$ and antibody against $\beta$-actin $(1: 5000)$ were purchased from ProteinTech Group, Inc. (Chicago, IL, USA). Anti-ERK1/2 (1:1000), anti-phospho-ERK1/2 (1:1000) and phosphorylated ERK1/2 inhibitor, PD98059 were purchased from Cell Signaling Technology, Inc. (Shanghai, China). Anti-maspin (1:1000), anti-Bax $(1: 1000)$, anti- $\alpha$-tubulin $(1: 5000)$ and anti-Bcl-2 $(1: 500)$ were purchased from Sangon, Ltd (Shanghai, China). Recombinant caspase-3 was purchased from Sino Biological Inc. (Beijing, China). MTS assay kit was purchased from Promega Company (Madison, WI, USA).

\section{Cell culture}

Two human NSCLC cell lines (A549 and SK-1) were purchased from the Cell Resource Center, Peking Union Medical College (CRC/PUMC, National Sciences \& Technology Infrastructure (Beijing, China)). Cells were incubated in DMEM/F-12 culture medium with $10 \% \mathrm{FBS}, 100 \mathrm{U} / \mathrm{ml}$ penicillin and streptomycin at $37^{\circ} \mathrm{C}$ in a humidified incubator containing $5 \% \mathrm{CO}_{2}$.

\section{Morphology assay}

Cells at $70 \%$ confluence were exposed to SFN-Cys at a series of concentrations: $0,10,20,30,40$ and $50 \mu \mathrm{M}$ for $24 \mathrm{~h}$ in a $6-\mathrm{cm}$ dish. Cell morphology was viewed with phase contrast microscope at $\times 100$ magnification (Leica, Wetzlar, Germany). Digital camera was connected to record cell morphology.

\section{Cell viability assay}

Cell viability was evaluated via MTS assay kit as described in the specifications (Madison, WI, USA). Cells $\left(4 \times 10^{3}\right)$ were seeded into a Corning Carbo-BIND 96-well plate and each well was added with a corresponding dose of SFN-Cys for $24 \mathrm{~h}$. Next, $20 \mu \mathrm{l}$ of MTS reagent was added to each well and incubated at $37^{\circ} \mathrm{C}$ for $1 \mathrm{~h}$. The absorbance values were tested at $490 \mathrm{~nm}$ on a BioTek Synergy HT Multi-Detection Microplate Reader (BioTek, Winooski, VT, USA).

\section{Apoptosis assay}

Apoptosis was determined by an Annexin V-fluorescein isothiocyanate (FITC)/propidium iodide (PI) assay. After the SFN-Cys treatment, cells were harvested and resuspended in $100 \mu \mathrm{l}$ binding buffer to reach a concentration of $1 \times 10^{6}$ cells/ml. Further, $5 \mu \mathrm{l}$ Annexin V-FITC and $5 \mu \mathrm{l} \mathrm{PI}$ $(20 \mu \mathrm{g} / \mathrm{ml})$ were added to the cells, which were incubated for $15 \mathrm{~min}$ in the dark. A total of $400 \mu \mathrm{l}$ binding buffer was added to each tube, and the cells were tested via the BD LSRFortessa flow cytometer (Becton, Dickinson and Company, San Jose, CA, USA). These data were analyzed by WinMDI version 2.9 software (Purdue University Cytometry Laboratories, West Lafayette, IN, USA).

\section{Western blot}

The treated cells were collected and lysed with lysis buffer $(25 \mathrm{mM}$ Tris- $\mathrm{HCl}$, $150 \mathrm{mM} \mathrm{NaCl}, 1 \mathrm{mM}$ EDTA, 1\% NP-40, 5\% glycerol, pH 7.4), the cell lysates were centrifuged at $12500 \times g$ for $15 \mathrm{~min}$. Equal amount of proteins were separated via $10 \%$ SDS-PAGE gels and transferred to nitrocellulose membranes. The membranes were blocked with $1.5 \%$ BSA for $1 \mathrm{~h}$ and subsequently incubated with the corresponding primary antibodies at $4{ }^{\circ} \mathrm{C}$ for $12 \mathrm{~h}$ ( $\alpha$-tubulin and $\beta$-actin were incubated at room tempreture for $30 \mathrm{~min}$ ). After incubation, the membranes were washed with phosphatebuffered saline with Tween $20(0.05 \%)$, and then incubated by the fluorescence-labeled secondary antibody (LI-COR Biosciences, Lincoln, NE, USA) for $1 \mathrm{~h}$ at room temperature. After washing, the protein bands were detected through the Odyssey Infrared Imaging System (LI-COR Biosciences). $\beta$-Actin was used as an internal control.

\section{Recombinant caspase-3 cleavage assay}

A549 and SK-1 cells were incubated with $20 \mu \mathrm{M}$ SFN-Cys for $24 \mathrm{~h}$ and the collected cells were lysed in $25 \mathrm{mM}$ Tris- $\mathrm{HCl}, 150 \mathrm{mM} \mathrm{NaCl}, 1 \mathrm{mM}$ EDTA, 1\% NP-40, 5\% glycerol, pH 7.4 and $24 \mu \mathrm{g}$ protein was incubated with $10 \mu \mathrm{l}$ recombinant caspase-3 (Sino Biological Inc.) in $50 \mu \mathrm{l}$ reaction buffer containing $25 \mathrm{mM}$ Hepes $\mathrm{pH} 7.5,0.1 \%(\mathrm{w} / \mathrm{v})$ Chaps, $10 \mathrm{mM} \mathrm{DTT}$, at $37^{\circ} \mathrm{C}$ for $6 \mathrm{~h}$. After incubation, western blot analysis was used to detect $a$-tubulin.

\section{Immunofluorescence assay}

The cells $\left(4 \times 10^{4}\right)$ were incubated in a 24-well dish for $10 \mathrm{~h}$ first and then treated with $20 \mu \mathrm{M} \mathrm{SFN-Cys} \mathrm{for} 24 \mathrm{~h}$. These cells were fixed with $4 \%$ paraformaldehyde for $20 \mathrm{~min}$ and permeabilized with $0.5 \%$ Triton X-100 for $15 \mathrm{~min}$ at room temperature. After blocking by $5 \%$ BSA for $30 \mathrm{~min}$, the cells were incubated with primary antibodies $(1: 50)$ for $2 \mathrm{~h}$ and incubated with the fluorescence-labeled secondary antibody $(1: 500)$ for $1 \mathrm{~h}$. The glass coverslips were stained with DAPI and the images were viewed on confocal laser scanning microscope (Olympus FV1000; Olympus Corp., Tokyo, Japan).

\section{Statistical analysis}

Data are calculated as the mean \pm S.D. and statistical differences were analyzed by ANOVA followed by Student's $t$-test. All statistical analyses were done through SPSS 18.0 software package (International Business Machines Corporation, Armonk, NY, USA).

\section{ACKNOWLEDGEMENTS}

This study was supported by the National Natural Science Foundation of China (grant numbers 81272843 and 81601993 ). All authors participated in the writing and review of the manuscript and accepted full responsibility for the overall content. The funders had no role in the study design, data collection and analysis, decision to publish or preparation of the manuscript.

\section{COMPETING INTERESTS}

The authors declare no conflict of interest.

\section{REFERENCES}

1 Thorsteinsson H, Alexandersson A, Oskarsdottir GN, Skuladottir R, Isaksson HJ, Jonsson $\mathrm{S}$ et al. Resection rate and outcome of pulmonary resections for non-small-cell lung cancer: a nationwide study from Iceland. J Thorac Oncol 2012; 7: 1164-1169.

2 Zhang BY, Wang YM, Gong H, Zhao H, Lv XY, Yuan GH et al. Isorhamnetin flavonoid synergistically enhances the anticancer activity and apoptosis induction by cis-platin and carboplatin in non-small cell lung carcinoma (NSCLC). Int J Clin Exp Pathol 2015; 8: 25-37.

3 Wiczk A, Hofman D, Konopa G, Herman-Antosiewicz A. Sulforaphane, a cruciferous vegetable-derived isothiocyanate, inhibits protein synthesis in human prostate cancer cells. Biochim Biophys Acta 2012; 1823: 1295-1305.

4 Clarke JD, Hsu A, Williams DE, Dashwood RH, Stevens JF, Yamamoto M et al. Metabolism and tissue distribution of sulforaphane in Nrf2 knockout and wildtype mice. Pharm Res 2011; 28: 3171-3179.

5 Dinkova-Kostova AT, Kostov RV. Glucosinolates and isothiocyanates in health and disease. Trends Mol Med 2012; 18: 337-347.

6 Myzak MC, Karplus PA, Chung FL, Dashwood RH. A novel mechanism of chemoprotection by sulforaphane: inhibition of histone deacetylase. Cancer Res 2004; 64: 5767-5774.

7 Feng Y, Niu LL, Wei W, Zhang WY, Li XY, Cao JH et al. A feedback circuit between miR-133 and the ERK1/2 pathway involving an exquisite mechanism for regulating myoblast proliferation and differentiation. Cell Death Dis 2013; 4: e934.

8 Tong WG, Ding XZ, Talamonti MS, Bell RH, Adrian TE. LTB4 stimulates growth of human pancreatic cancer cells via MAPK and PI-3 kinase pathways. Biochem Biophys Res Commun 2005; 335: 949-956.

9 Lee WJ, Hsiao M, Chang JL, Yang SF, Tseng TH, Cheng CW et al. Quercetin induces mitochondrial-derived apoptosis via reactive oxygen species-mediated ERK activation in HL-60 leukemia cells and xenograft. Arch Toxicol 2015; 89: 1103-1117.

10 Bodenstine TM, Seftor RE, Khalkhali-Ellis Z, Seftor EA, Pemberton PA, Hendrix MJ. Maspin: molecular mechanisms and therapeutic implications. Cancer Metastasis Rev 2012; 31: 529-551.

11 Ates U, Gollu G, Kucuk G, Billur D, Bingol-Kologlu M, Yilmaz Y et al. Increase in proapoptotic Bax expression and decrease in anti-apoptotic Bcl-2 expression in newborns with necrotizing enterocolitis. Arch Argent Pediatr 2016; 114: 243-247. 
12 Hamacher-Brady A, Brady NR. Bax/Bak-dependent, Drp1-independent targeting of $X$-linked Inhibitor of apoptosis protein (XIAP) into inner mitochondrial compartments counteracts Smac/DIABLO-dependent effector caspase activation. J Biol Chem 2015; 290: 22005-22018.

13 Bhujade A, Gupta G, Talmale S, Das SK, Patil MB. Induction of apoptosis in A431 skin cancer cells by Cissus quadrangularis Linn stem extract by altering Bax-Bcl-2 ratio, release of cytochrome $c$ from mitochondria and PARP cleavage. Food Funct 2013; 4: 338-346.

14 Parsons MJ, Fassio SR, Bouchier-Hayes L. Detection of initiator caspase induced proximity in single cells by caspase bimolecular fluorescence complementation. Methods Mol Biol 2016; 1419: 41-56.

15 Yang B, Li L, Pu F, You W, Huang H, Ke C. Molecular cloning of two molluscan caspases and gene functional analysis during Crassostrea angulata (Fujian oyster) larval metamorphosis. Mol Biol Rep 2015; 42: 963-975.

16 Pai HC, Kumar S, Shen CC, Liou JP, Pan SL, Teng CM. MT-4 suppresses resistant ovarian cancer growth through targeting tubulin and HSP27. PLOS ONE 2015; 10: e0123819.

17 Tang D, Wang C, Gao Y, Pu J, Long J, Xu W. Deep hypothermia-enhanced autophagy protects PC12 cells against oxygen glucose deprivation via a mitochondrial pathway. Neurosci Lett 2016; 632: 79-85.

18 Mashimo M, Kato J, Moss J. ADP-ribosyl-acceptor hydrolase 3 regulates poly (ADPribose) degradation and cell death during oxidative stress. Proc Natl Acad Sci USA 2013; 110: 18964-18969.

19 Mao Y, Zhang J, Hou L, Cui X. The effect of beta-elemene on alpha-tubulin polymerization in human hepatoma HepG2 cells. Chin J Cancer Res 2013; 25: 770-776.

20 Isshiki K, Hirase T, Matsuda S, Miyamoto K, Tsuji A, Yuasa K. Death-associated protein kinase 2 mediates nocodazole-induced apoptosis through interaction with tubulin. Biochem Biophys Res Commun 2015; 468: 113-118.

21 Li C, Zhou Y, Peng X, Du L, Tian H, Yang G et al. Sulforaphane inhibits invasion via activating ERK1/2 signaling in human glioblastoma U87MG and U373MG cells. PLOS ONE 2014; 9: e90520.

22 Lan F, Pan Q, Yu H, Yue X. Sulforaphane enhances temozolomide-induced apoptosis because of down-regulation of miR-21 via Wnt/beta-catenin signaling in glioblastoma. J Neurochem 2015; 134: 811-818.

23 Gasper AV, Al-Janobi A, Smith JA, Bacon JR, Fortun P, Atherton C et al. Glutathione S-transferase M1 polymorphism and metabolism of sulforaphane from standard and high-glucosinolate broccoli. Am J Clin Nutr 2005; 82: 1283-1291.

24 Tian H, Zhou Y, Yang G, Geng Y, Wu S, Hu Y et al. Sulforaphane-cysteine suppresses invasion via downregulation of galectin-1 in human prostate cancer DU145 and PC3 cells. Oncol Rep 2016; 36: 1361-1368.

25 Sun X, Deng QF, Liang ZF, Zhang ZQ, Zhao L, Geng $\mathrm{H}$ et al. Curcumin reverses benzidine-induced cell proliferation by suppressing ERK1/2 pathway in human bladder cancer T24 cells. Exp Toxicol Pathol 2016; 68: 215-222.
26 Wang X, Yu Z, Zhou Q, Wu X, Chen X, Li J et al. Tissue transglutaminase-2 promotes gastric cancer progression via the ERK1/2 pathway. Oncotarget 2016; 7: 7066-7079.

27 Zhang W, Shi HY, Zhang M. Maspin overexpression modulates tumor cell apoptosis through the regulation of $\mathrm{Bcl}-2$ family proteins. BMC Cancer 2005; 5: 50.

28 Dzinic SH, Chen K, Thakur A, Kaplun A, Bonfil RD, Li X et al. Maspin expression in prostate tumor elicits host anti-tumor immunity. Oncotarget 2014; 5: 11225-11236.

29 Huang CY, Chang YJ, Luo SD, Uyanga B, Lin FY, Tai CJ et al. Maspin mediates the gemcitabine sensitivity of hormone-independent prostate cancer. Tumour Biol 2016; 37: 4075-4082.

30 Lee SJ, Jang H, Park C. Maspin increases Ku70 acetylation and Bax-mediated cell death in cancer cells. Int J Mol Med 2012; 29: 225-230.

31 Hennessy EJ. Selective inhibitors of $\mathrm{Bcl}-2$ and $\mathrm{BCl}-\mathrm{xL}$ : balancing antitumor activity with on-target toxicity. Bioorg Med Chem Lett 2016; 26: 2105-2114.

32 Rahman MA, Shirai M, Aziz MA, Ushirokita R, Kubota S, Suzuki H et al. An epistatic effect of apaf-1 and caspase-9 on chlamydial infection. Apoptosis 2015; 20: 1271-1280.

33 Wurstle ML, Rehm M. A systems biology analysis of apoptosome formation and apoptosis execution supports allosteric procaspase-9 activation. J Biol Chem 2014; 289: 26277-26289.

34 Wu H, Che X, Zheng Q, Wu A, Pan K, Shao A et al. Caspases: a molecular switch node in the crosstalk between autophagy and apoptosis. Int J Biol Sci 2014; 10: 1072-1083.

35 Singh BK, Tripathi M, Chaudhari BP, Pandey PK, Kakkar P. Natural terpenes prevent mitochondrial dysfunction, oxidative stress and release of apoptotic proteins during nimesulide-hepatotoxicity in rats. PLOS ONE 2012; 7: e34200.

36 Zhang YZ, Chen X, Fan XX, He JX, Huang J, Xiao DK et al. Compound library screening identified cardiac glycoside digitoxin as an effective growth inhibitor of gefitinib-resistant non-small cell lung cancer via downregulation of alpha-tubulin and inhibition of microtubule formation. Molecules 2016; 21: 374.

37 Zhang F, Su B, Wang C, Siedlak SL, Mondragon-Rodriguez S, Lee HG et al. Posttranslational modifications of alpha-tubulin in alzheimer disease. Transl Neurodegener 2015; 4: 9.

This work is licensed under a Creative Commons Attribution 4.0 International License. The images or other third party material in this article are included in the article's Creative Commons license, unless indicated otherwise in the credit line; if the material is not included under the Creative Commons license, users will need to obtain permission from the license holder to reproduce the material. To view a copy of this license, visit http://creativecommons.org/licenses/ by/4.0/

(c) The Author(s) 2017 